\title{
Raman scattering and photoluminescence study of porous silicon formed on n-type silicon
}

\author{
S K DEB, NEELU MATHUR, A P ROY, S BANERJEE ${ }^{\dagger}$ and \\ A SARDESAI ${ }^{\dagger}$ \\ Solid State Physics Division, Bhabha Atomic Research Centre, Bombay 400085, India \\ ${ }^{\dagger}$ Tata Institute of Fundamental Research, Homi Bhabha Road, Bombay 400005 , India
}

\begin{abstract}
We report Raman scattering and photoluminescence studies on porous silicon film formed on n-type silicon. The Raman spectra over the sample surface exhibit considerable variation whereas the photoluminescence spectra are practically identical. Our results indicate that, well inside the film surface, it consists of spherical nanocrystals of typical diameter $\approx 100 \AA$, while on the edge these nanocrystals are $\geqslant 300 \AA$. We further observe that there is no correlation between the photoluminescence peak position and the nanocrystal diameter. This suggests that the origin of the photoluminescence is due to radiative recombination between defect states in the bulk as well as on the surface of the nanocrystal.
\end{abstract}

Keywords. Porous silicon; Raman scattering; photoluminescence.

\section{Introduction}

Porous silicon (PS-Si) has been prepared and studied for many years (Uhlir 1956). The principal interest was its application in Si-on-insulators technology (Imai 1981). Recently there has been a spurt in activity in the study of optical properties of this material due to its efficient room-temperature photoluminescence (PL)(Canham 1990) and electroluminescence (EL) (Halimaoui et al 1991) with the hope of its possible use in Si-based optoelectronic devices. But there is considerable controversy over the origin of this spectacular property. Different techniques are being employed to study the properties of PS-Si to understand this phenomenon. Among the various mechanisms, models based on the quantum confinement (Lehmann and Gosele 1991) of charge carriers and those based on chemical species like siloxene (Brandt et al 1992), Si hydrides (Tsai et al 1992) and polysilane (Prokes et al 1992) are the most controversial in nature. Raman spectroscopy is one of the techniques which can be employed to distinguish between these mechanisms and in fact the origin of the mechanism based on siloxene (Brandt et al 1992) can be traced back to Raman spectroscopic study of porous silicon and a few chemical compounds of the type $\mathrm{Si}: \mathrm{O}_{\boldsymbol{x}}: \mathrm{H}_{\boldsymbol{y}}$. For the quantum confinement model it is necessary to determine the size of the nanostructures on PS-Si films and correlate them with the intensity and peak position of the PL. Raman spectroscopy has earlier been successfully used to determine the size of nanostructures (Campbell and Fauchet 1986) in amorphous $\mathrm{Si}$ and other materials and is also currently being used for PS-Si films (Sui et al 1992). Its advantage over other techniques (i.e. TEM) is that it is non-destructive and both Raman and PL spectra can be obtained from the same region of the sample using identical experimental geometry, facilitating correlation between the PL spectra and nanostructure size (Sood et al 1992).

Most of the Raman scattering studies of PS-Si in the literature are for films formed on p-type $\mathrm{Si}$, but there is hardly any published data on those prepared using n-type 
Si. Although the presence of holes is essential for PS-Si formation it is known that PS-Si films can be prepared on n-type material under illumination and they also exhibit similar photoluminescence. But it has been shown that depending on the conductivity of the $\mathrm{Si}$ substrate, the type and size of microstructures on n-type materials can be quite different from those on p-type materials (Smith and Collins 1992). We report here Raman scattering investigations carried out on PS-Si films prepared on n-type materials and the determined sizes of the nanostructures present. We further measure the PL spectra from the same sample region to examine any possible correlation between the Raman and PL spectra.

\section{Experimental}

Porous silicon films on n-type $\mathrm{Si}(001)$ wafers $(4-6 \Omega$-cm resistivity) were prepared by electrochemical etching in $4.8 \% \mathrm{HF}$ at $\approx 20 \mathrm{~mA} / \mathrm{cm}^{2}$ under illumination from a tungsten lamp. The anodization time was $50 \mathrm{~min}$. The samples were stored at ambient conditions for over a year before Raman spectra were recorded. The thickness of the film is $>5 \mu \mathrm{m}$.

Raman spectra were excited using $20 \mathrm{~mW}$ of the $4880 \AA$ line from an $\mathrm{Ar}^{+}$laser (Innova-70) focussed to a spot size of $50 \mu \mathrm{m}$. The Raman scattered light was collected in back-scattering geometry by a f1-2 lens and focussed on a double monochromator (Roy and Bansal 1988) with a cooled ITT FW 130 photomultiplier tube (PMT) followed by a photon counting system. With such a system the spectra can be stored digitally for further processing. PL spectra from the same region can be collected at right angles and recorded using a compact PL set-up consisting of a Jobin-Yvon H20 UV monochromator, a multialkali PMT (Hamamatsu R 1477) and a lock-in detection system. This is shown schematically in figure 1. The PL spectra shown in

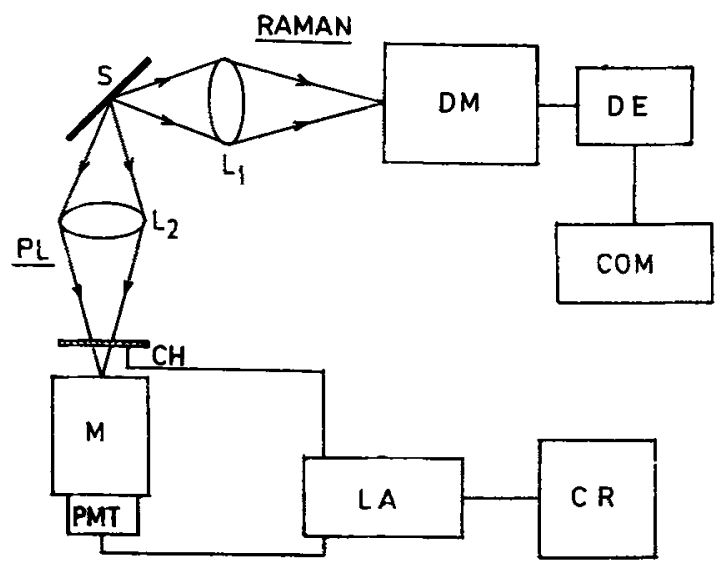

Figure 1. Schematic diagram of the Raman and PL spectroscopy set-up. $S$ sample; $L_{1}$ and $\mathrm{L}_{2}$, collection lenses; $\mathrm{DM}$, double monochromator; $\mathrm{DE}$, detection electronics; $C O M$, computer; $\mathbf{C H}$, chopper; $\mathrm{M}$, monochromator; PMT, photomultiplier tube; LA, lock-in amplifier; $\mathbf{C R}$, chart recorder. 
the figures are as-recorded ones without correction for monochromator and PMT response. The wafers were oriented such that the polarization of the incident radiation was along the $X$-axis where $X, Y$ and $Z$ denote the $(001),(010)$ and $(001)$ crystallographic directions respectively.

\section{Theory}

A Raman spectrum taken on a perfect single crystal consists of optical phonons close to the Brillouin zone centre due to the $q \approx 0$ selection rule and the spectrum is a symmetric line with a FWHM which can be related to the phonon lifetime. But in nanocrystalline materials, the phonons are spatially confined, which leads to the breakdown of the $q \approx 0$ selection rule. This results in contribution from other $q$ points and depending on the dispersion of the particular phonon branch, the line shape becomes asymmetric with a shift in the peak position (Richter et al 1981). The exact nature of the phonon confinement is still being debated and the relation between the size of the nanostructures and the line shape depends on the particular form of confinement function used (Campbell and Fauchet 1986). For simplicity the phonons are assumed to be confined to either spherical quantum dots or cylindrical quantum wires. The most commonly employed confinement function is of the type $\exp \left(-8 \pi^{2} r^{2} / L^{2}\right)$, where $L$ is the diameter of the spherical dot or that of the cylindrical wire. The detailed line shapes for both the cases have been worked out in the literature and are given by

$$
I(\omega)=\text { const. } \int \mathrm{d}^{3} q \exp \left[-q^{2} L^{2} / 4 /\left[\left\{\omega-\omega_{0}(q)\right\}^{2}+\left(\Gamma_{0} / 2\right)^{2}\right]\right],
$$

where $q$ is in units of $(2 \pi / c), L$ in units of $c$, and $c$ is the lattice constant of $\mathrm{Si}(=0.54 \mathrm{~nm})$. The appropriate $\mathrm{d}^{3} q$ is chosen for sphere or cylinder. $\omega_{0}(q)$ denotes the phonon frequency at point $q$ of the Brillouin zone and $\Gamma_{0}$ is the FWHM at $q=0$. For crystalline silicon, the first-order Raman spectrum consists of a triply degenerate optical phonon at $\approx 519 \mathrm{~cm}^{-1}$ with a $\Gamma_{0} \approx 2.8 \mathrm{~cm}^{-1}$. For backscattering from a (001) face only the LO component can be observed in the $Z(X Y) V$ geometry. The dispersion of the phonon branch has been determined by Dolling (1963) and can be fitted with a parabolic dispersion relation of the type $\omega_{0}(q)=\omega_{0}-A q^{2}$. From the experimental data the value of $A$ determined is $\approx 140 \mathrm{~cm}^{-1}$. A curve relating the FWHM, peak shift and nanocrystal diameter can be obtained for both the cases and can be employed immediately to obtain the size and type of the nanostructure (Campbell and Fauchet 1986).

\section{Results and analysis of data}

The PL and Raman spectra for several points over the surface of the PS-Si film have been recorded. Although the PL spectra from different points are more or less identical in peak position and width, there is considerable difference in the Raman spectra. The PL spectra (as-recorded) obtained from the edge and from well inside the PS-Si film are shown in figure 2. The peak position and width of the PL spectra for both the cases are $\approx 680 \mathrm{~nm}$ and $120 \mathrm{~nm}$ respectively. The Raman spectra from the same 


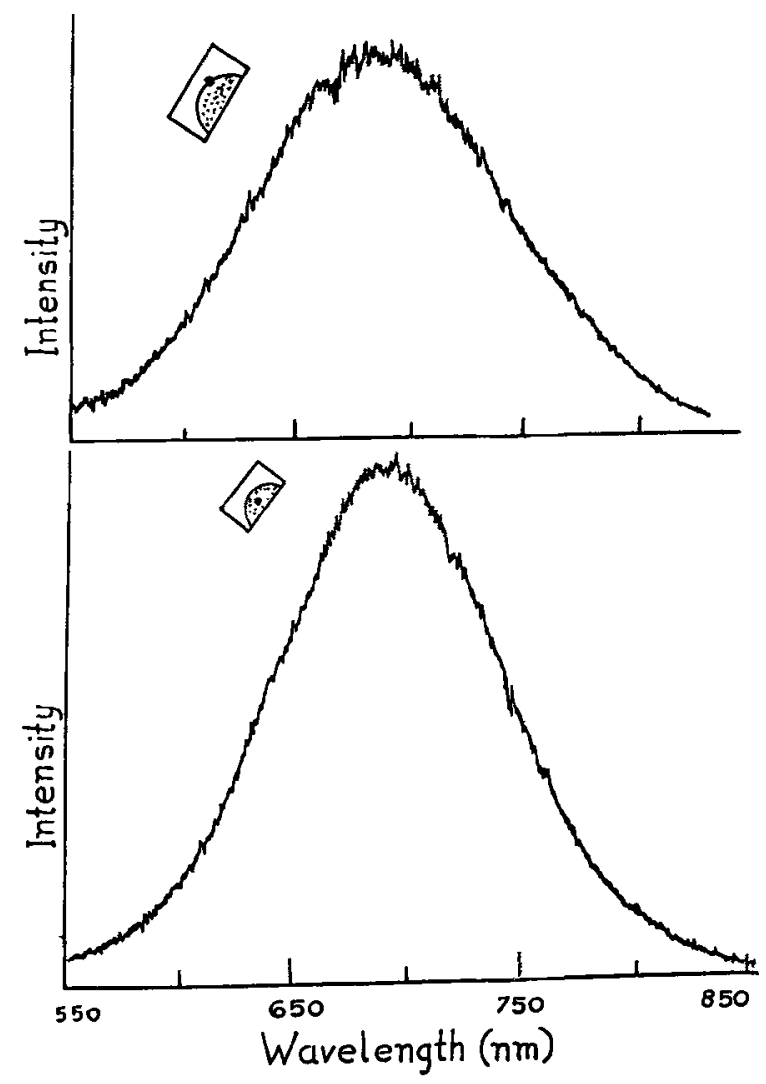

Figure 2. PL spectra from the edge and inside of the PS-Si film. The small dots denote the film and the big dot ( $(\bullet)$ indicates the position of the exciting-beam spot.

points on the edge and from well inside the film are shown in figure 3 . The line shape for the crystalline part $(c-\mathrm{Si})$ is also shown in the same figure for comparison. It can be seen that the Raman line shape from the edge is almost identical to that for crystalline silicon (c-Si) whereas that from well inside the sample is asymmetric with a low-frequency tail and a shift in the peak position towards lower Raman shift. Raman spectra for different points on the sample have been obtained and the experimental FWHM and peak shift (with respect to the $c$-Si peak) have been determined from these spectra and are shown in figure 4 . We note that the FWHM is comparable to the instrument resolution $\left(\approx 1.8 \mathrm{~cm}^{-1}\right)$ and hence in order to compare the theoretical and experimental values we calculate the line shape by convoluting the instrument resolution function with the line shape given in (1). The instrumental line shape can be obtained from the shape of the $528 \mathrm{~cm}^{-1}$ plasma line recorded along with the Raman spectrum. A Gaussian form has been fitted to this line shape and this has been used in the convolution to obtain the final line shape. Similarly the peak shift also can be determined very accurately from the position of the same plasma line and the accuracy is $\pm 0.5 \mathrm{~cm}^{-1}$. The FWHM and peak shift obtained from the calculated line profile for different values of the diameters are 


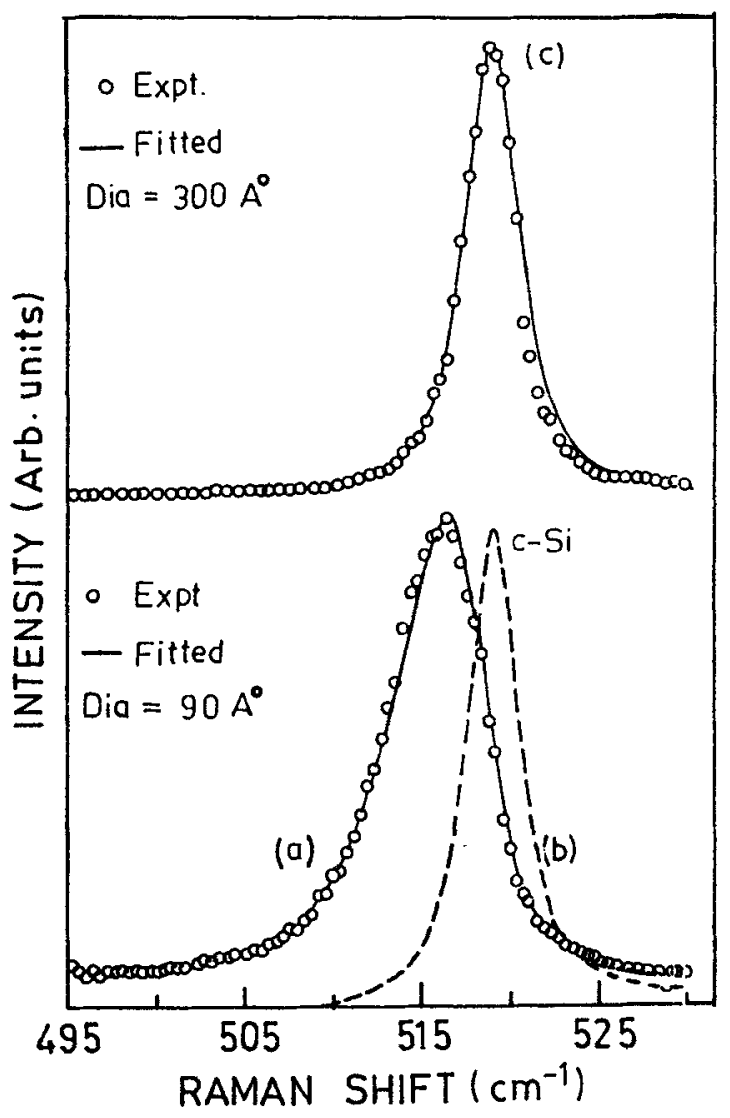

Figure 3. Raman spectra of PS-Si film from the points shown in figure 2[(a) from well inside and (c) from the edge]. The crystalline-silicon (c-Si) line is also shown in (b) for comparison. The continuous line is the fitted curve for spherical nanoparticles of diameters shown.

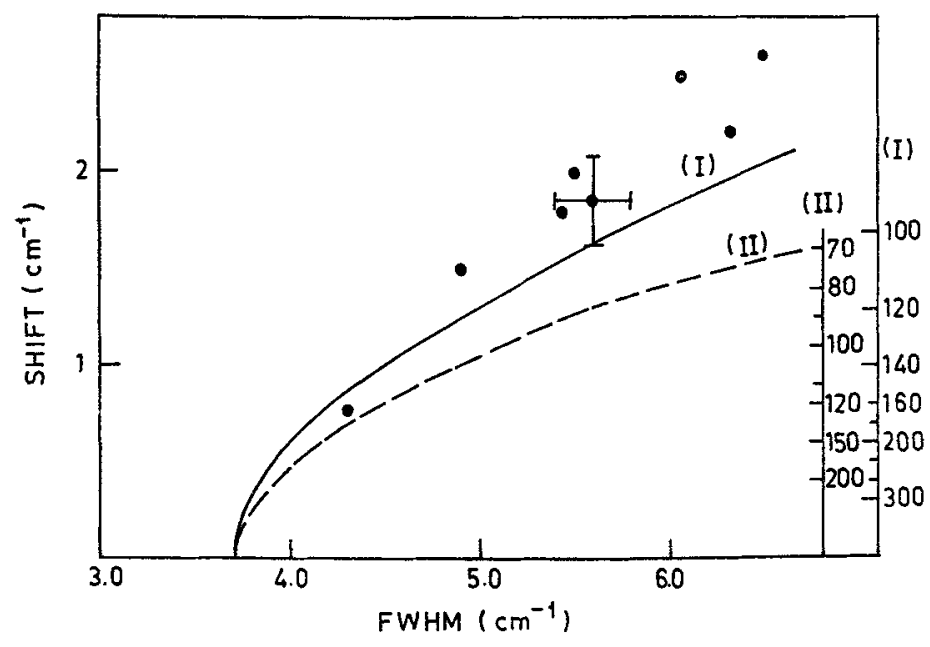

Figure 4. The peak shift and FWHM of Raman spectra obtained from different regions of the film. The experimental errors in these quantities are also shown. The continuous (I) and dashed (II) curves are calculated values for diameters indicated on the right. 
shown in figure 4 by continuous curve for spherical dots and by dashed line for cylinders. Munder et al (1992) included the effect of strain present in the PS-Si film to analyse the Raman spectra and have shown that there is an additional shift of the peak position towards lower frequency. It can be seen that the FWHM and peak shift for different regions over the sample surface are different, and by comparing them with calculated values it can be seen that the agreement is better with spherical nanoparticles rather than with cylindrical wires.

The size of the quantum dots can be obtained by fitting the theoretical line profile (as calculated using (1) and convoluting with the resolution function) to the experimental data. These are shown in figure 3. The agreement is excellent and for a region well inside the film the particle size obtained is $\approx 90 \AA$. On the other hand a line shape obtained from the film edge yields a particle size $\approx 300 \AA$. The difference in the sizes may be due to the particular geometry of the electrochemical cell used for preparation of PS-Si film from n-type material, under illumination. The edges of the film may receive lower intensity of radiation due to shadowing from the sides leading to fewer photo-generated holes and hence larger dimension of the particles.

We have also obtained Raman spectra from the film in the polarized $\{Z(X X) \bar{Z}\}$ and depolarized $\{Z(X Y) \bar{Z}\}$ geometries and we observe that they are more or less identical in width and intensity. This can be explained by a random orientation of the particles, because it is known that the Raman spectrum from $\mathrm{Si}(100)$ face has strong polarization dependence whereas those from $\mathrm{Si}(111)$ face is polarizationindependent.

\section{Discussion}

We observe that the dimension of nanocrystals formed well inside the sample is $\approx 100 \AA$ while those near the edge are $\geqslant 300 \AA$. It is generally observed that for p-type samples the dimensions are much smaller $(\approx 30-50 \AA$ ) (Munder et al 1992; Sui et al 1992) and the observed peak shift and FWHM are much larger. As reported earlier (Sood et al 1992; Lee et al 1993) we also do not observe any apparent correlation between nanocrystal size and PL peak position. If the carriers are confined in the nanoparticles and the PL is assumed to arise from recombination of band edge photoexcited charge carriers, then the PL peak position should shift towards shorter wavelength for smaller size of dots. This is contrary to our observation. Lee et al (1993) reported similar drastic variation in Raman spectra over the surface of PS-Si film formed on p-type material, but they observed reduction in FWHM and peak shift for regions well inside the sample while the spectrum from the edge was much broader with larger peak shift. This is opposite to what we observe. They have also reported that the PL spectra do not change for different regions of the sample. Their observation has been explained to arise from the sample preparation procedure. Electronic structure calculation for quantum dots of different diameters shows that (Proot et al 1992) the band gap for dots with diameters $\geqslant 100 \AA$ is practically the same as that for bulk silicon. Hence PL spectra with peak position at $\approx 680 \mathrm{~nm}$ cannot arise from band edge radiative recombination of carriers in these quantum dots. As regards the origin of PL from chemical species like siloxene and its different derivatives, it is well known that their PL spectra can be changed considerably by heat treatment or by oxidation (Brandt et al 1992). But it should also be noted that (Stutzmann et al 1992) there are corresponding changes in the Raman spectra as well. 
Taking this into account, if we assume that a particular chemical species is responsible for the PL from the edge as well as from inside the film surface then the Raman spectrum also should have been identical. This is not supported by our observations. We would like to add that, although the microstructure for low-doped n-type samples are column-like, the Raman spectroscopy does not corroborate this and in fact supports the fact that the particulate nature is more important for the luminescence and not 'wire'-like structures.

\section{Conclusion}

Raman spectra from photoluminescent PS-Si film formed on n-type Si exhibit presence of nanocrystalline spheres with random orientations. The diameters for regions well inside the sample is $\cong 100 \AA$ while those on edge $\geqslant 250 \AA$. However the PL spectra do not show any difference, which indicates that PL does not arise from band edge recombination in these nanocrystallites. On the other hand it also does not agree with the chemical species scenario. Our observation seems to agree more with the currently held view that the photoexcitation takes place in the nanocrystals but the PL arises from recombination taking place between the defect-related states in the bulk as well as on the surface of the nanoparticles (Koch 1993).

\section{Acknowledgements}

We thank Prof. B M Arora for useful discussion and Drs B A Dasannacharya and K R Rao for constant encouragement.

\section{References}

Brandt M S, Fuchs H D, Stutzmann M, Weber J and Cardona M 1992 Solid State Commun. 81307

Campbell I H and Fauchet P M 1986 Solid State Commun. 58739

Canham L T 1990 Appl. Phys. Lett. 571046

Dolling G 1963 Inelastic neutron scattering from solids and liquids (Vienna: IAEA) Vol. 11 pp 37-48

Halimaoui A, Oules C, Bomchil G, Bsiesy A, Gaspard F, Herino R, Ligeon M and Muller F 1991 Appl. Phys. Lett. 59304

Imai K 1981 Solid State Electron. 24159

Koch F 1993 Preprint, MRS Meeting, USA

Lee H J et al 1993 Appl. Phys. Lett. 62855

Lehmann V and Gosele U 1991 Appl. Phys. Lett. 58856

Munder H, Andrzejak C, Berger M G, Klemradt U, Luth H, Herino R and Ligeon M 1992 Thin Solid Films 22127

Prokes S M, Glembocki O J, Bermudez V M. Kaplan R, Fridersdorf L E and Searson P C 1992 Phys. Rev. B45 13788

Proot J P, Delerue C and Allan G 1992 Appl. Phys. Lett. 611948

Richter H, Wang Z P and Ley L 1981 Solid State Commun. 39625

Roy A P and Bansal M L 1988 Indian J. Pure Appl. Phys. 26218

Smith R L and Collins S D 1992 J. Appl. Phys. 71 R1

Sood A K, Jayaram K and Muthu D V S 1992 J. Appl. Phys. 724963

Stutzmann M, Weber J, Brandt M S, Fuchs H D, Rosenbauer M, Deak P, Hopner A and Breitschwerdt A 1992 Adv. Solid State Phys. 32179

Sui Z, Leong P P, Herman I P, Higashi G S and Temkin H 1992 Appl. Phys. Lett. 502086

Tsai C et al 1992 Appl. Phys. Lett. 601700

Uhlir A 1956 Bell. Syst. Tech. J. 35333 